\title{
Formulation of stellar speckle interferometry in terms of joint spatial/spatial-frequency representations
}

\author{
Rafael Navarro \\ Instituto de Optica, Serrano 121, 28006 Madrid, Spain \\ Antonio F. Pérez-Rendón \\ Instituto de Astrofísica de Canarias, 38200 La Laguna, Tenerife, Spain
}

Received March 22, 1990; accepted September 24, 1990

\begin{abstract}
We propose a generalized formulation of stellar speckle interferometry (SSI) and related techniques in terms of joint spatial/spatial-frequency energy representations. We show how, within this formulation, techniques such as that of Knox-Thompson, or even standard SSI, appear to be particular implementations of a more general method. This generalized SSI is derived in a straightforward manner from the proposed joint formulation. Results from a computer simulation are presented.
\end{abstract}

Stellar speckle interferometry (SSI) is a common technique used in astronomy to attain the diffraction-limit resolution of large ground-based telescopes. ${ }^{1,2}$ Let $i_{t}(x)$ be the image of the object $o(x)$ at time $t$; then

$$
i_{t}(x)=o(x) \otimes h_{t}(x),
$$

where $\otimes$ denotes convolution and $h_{t}(x)$ is the instantaneous point-spread function of the image-forming system: atmosphere-telescope. Conventional astronomical imaging consists of integrating Eq. (1) during a given exposure time. The resulting image is proportional to the average $\left\langle i_{t}(x)\right\rangle=o(x) \otimes\left\langle h_{t}(x)\right\rangle$. Instead, SSI consists of averaging the power spectra of a set of short-exposure images,

$$
\left\langle\left|I_{t}(u)\right|^{2}\right\rangle=|O(u)|^{2}\left\langle\left|H_{t}(u)\right|^{2}\right\rangle \text {. }
$$

By applying the same averaging procedure to a reference point star it is possible to estimate $\left\langle\left|H_{t}(u)\right|^{2}\right\rangle$. Thus one could solve Eq. (2) to obtain $|O(u)|^{2}$ as far as the cutoff frequency of the telescope. Using this technique, one attains the diffraction limit at the cost of missing the phase of $O(u)$. Consequently one cannot reconstruct the object $o(x)$, since the Fourier transform (FT) of $|O(u)|^{2}$ gives the autocorrelation $Q(\Delta x)$ of the object, instead of $o(x)$. Several methods have been proposed to keep the information about the phase in SSI ${ }^{3-8}$ (see Ref. 9 for a useful review).

In this Letter we propose a formulation of SSI, and related techniques, based on joint energy spatial/spatial-frequency representations. From this powerful analytical framework ${ }^{10,11}$ some of the ideas involved in SSI can be generalized. Furthermore, one can find new and possibly more advantageous practical implementations. There are four basic functions in the joint representation: the product function $(\mathrm{PF})$, the Wigner distribution (WD), the ambiguity function (AF), and the spectral product (SP). It is easy to show that the four functions retain both high resolution and phase information. After that, we show how most of the speckle techniques appear in this formulation as particular implementations of a generalized SSI method. Finally, results of a computer simulation are presented.

Following Cohen, ${ }^{10}$ a general expression for a class of joint energy representations is given by ${ }^{11}$

$$
\begin{aligned}
& E_{i}(x, u ; \phi)=\frac{1}{(2 \pi)^{N}} \iiint_{-\infty}^{+\infty} \phi(\xi, \alpha) i^{*}(\rho-\alpha / 2) \\
& \quad \times i(\rho+\alpha / 2) \exp [2 \pi j(\xi x-\alpha u-\xi \rho)] \mathrm{d} \rho \mathrm{d} \xi \mathrm{d} \alpha,
\end{aligned}
$$

where $x, u, \xi, \alpha$, and $\rho$ are vectors in the general $N$ dimensional case. The function $\phi(\xi, \alpha)$ is a kernel that defines a particular representation. When $\phi(\xi, \alpha)=1$, then $E_{i}$ is the WD.11,12 The AF is also obtained by placing $\phi(\xi, \alpha)=2 \pi \delta(\alpha-x) \delta(\xi-u)$ in Eq. (3). It follows that the WD, along with its two single FT's (PF and SP) and with its double FT (AF), completes the simplest joint energy scheme. Figure 1 indicates the definitions and relations between the four basic functions of this scheme. Any function is computable from another by a single (adjacent in the diagram) or a

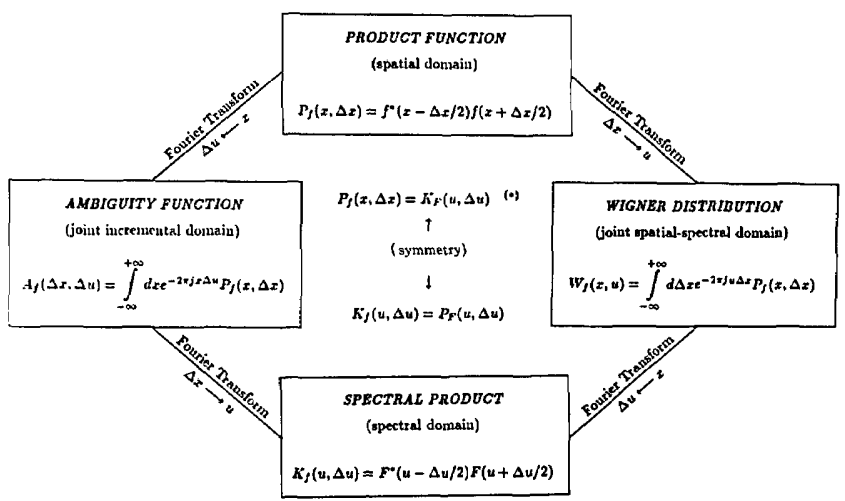

Fig. 1. Definitions and relations of the four basic functions of the joint energy representation scheme. The upper symmetry property $\left(^{*}\right)$ is only valid for real $f$. 
Table 1. Different Speckle Imaging Techniques in Terms of the Product Function and the Kernel Defining the Joint Representation ${ }^{a}$

\begin{tabular}{lll}
\hline \multicolumn{1}{c}{ Method } & \multicolumn{1}{c}{ Expression } & \multicolumn{1}{c}{ Kernel, $\phi(\xi, \alpha)$} \\
\hline Generalized SSI & $\left\langle P_{i}(x, \Delta x)\right\rangle$ & $A \delta(\xi-\Delta u), \Delta u<u_{c}$ \\
Standard SSI ${ }^{1,2}$ & $\int_{-\infty}^{+\infty} \mathrm{d} x\left\langle P_{i}(x, \Delta x)\right\rangle$ & $A \delta(\xi-\Delta u) \delta(\Delta u)$ \\
Knox-Thompson ${ }^{3}$ & $\mathrm{FT}\left\{\left\langle P_{i}(x, \Delta x)\right\rangle B\right\}_{\substack{x \rightarrow \Delta u \\
\Delta x \rightarrow u}}$ & $A \delta(\xi-\Delta u) B, \Delta u<u_{c}$ \\
Phase gradient $^{5}$ & $\delta(\Delta u) \mathrm{FT}\left\{x\left\langle P_{i}(x, \Delta x)\right\rangle B\right\}_{\substack{x \rightarrow \Delta u \\
\Delta x \rightarrow u}}$ & $A \alpha \delta(\xi-\Delta u) \delta(\Delta u) B$ \\
Exponential filtering $^{6}$ & $\delta(\Delta u) \mathrm{FT}\left\{e^{a x}\left\langle P_{i}(x, \Delta x)\right\rangle B\right\}_{\substack{x \rightarrow \Delta u \\
\Delta x \rightarrow u}}$ & $A e^{a \alpha} \delta(\xi-\Delta u) \delta(\Delta u) B$ \\
Triple correlation $^{4}$ & $\mathrm{FT}\left\{\left\langle i(\xi) \star P_{i}(\xi, \Delta x)\right\rangle_{(x)} B\right\}_{\substack{x \rightarrow \Delta u \\
\Delta x \rightarrow u}}$ & $A \delta(\xi-\Delta u) F_{i}^{*}(\Delta u) B$ \\
\hline
\end{tabular}

${ }^{a} A=2 \pi \delta(\alpha-x), B=\exp (2 \pi j x / 2 \Delta u)$

double (opposite) FT. On the other hand, the WD presents a set of interesting properties (see Refs. 11 and 12). Among these properties, the convolution theorem allows one to rewrite Eq. (1) in four different ways:

$$
\begin{aligned}
& P_{i}(x, \Delta x)=\left[P_{0}(\xi, \eta) \otimes P_{h}(\xi, \eta)\right]_{(x, \Delta x)}, \\
& W_{i}(x, u)=\left[W_{0}(\xi, u) \otimes W_{h}(\xi, u)\right]_{(x)}, \\
& A_{i}(\Delta x, \Delta u)=\left[A_{o}(\eta, \Delta u) \otimes A_{h}(\eta, \Delta u)\right]_{(\Delta x)}, \\
& K_{i}(u, \Delta u)=K_{o}(u, \Delta u) K_{h}(u, \Delta u) .
\end{aligned}
$$

Equations (4) indicate that the convolution in Eq. (1) becomes a double convolution (in $x$ and $\Delta x$ ) in the domain of the PF; it is a single convolution in both joint domains and a product in the domain of the SP.

In practice, there are two alternatives to implement SSI, one by averaging the power spectrum of shortexposure images and the other by averaging the autocorrelations. ${ }^{2}$ Both the power spectrum and autocorrelation are energy representations of the signal. In the joint scheme we can compute four equivalent averages:

$$
\begin{aligned}
\left\langle P_{i}(x, \Delta x)\right\rangle= & \left\langle i_{t}^{*}(x-\Delta x / 2) i_{t}(x+\Delta x / 2)\right\rangle \\
\left\langle W_{i}(x, u)\right\rangle= & \int_{-\infty}^{+\infty} \mathrm{d} \Delta x\left\langle P_{i}(x, \Delta x)\right\rangle \exp (-2 \pi j u \Delta x) \\
\left\langle A_{i}(\Delta x, \Delta u)\right\rangle= & \int_{-\infty}^{+\infty} \mathrm{d} x\left\langle P_{i}(x, \Delta x)\right\rangle \exp (-2 \pi j x \Delta u) \\
\left\langle K_{i}(u, \Delta u)\right\rangle= & \iint_{-\infty}^{+\infty} \mathrm{d} \Delta x \mathrm{~d} x\left\langle P_{i}(x, \Delta x)\right\rangle \\
& \times \exp [-2 \pi j(x \Delta u+u \Delta x)] \\
= & \left\langle I_{t}^{*}(u-\Delta u / 2) I_{t}(u+\Delta u / 2)\right\rangle
\end{aligned}
$$

These equations state that it does not matter which of the four functions one averages, since the FT of an average is the average of the FT. It is easy to show that the four averages of $P_{i}, W_{i}, A_{i}$, and $K_{i}$ retain both high resolution and phase information. That was dem-
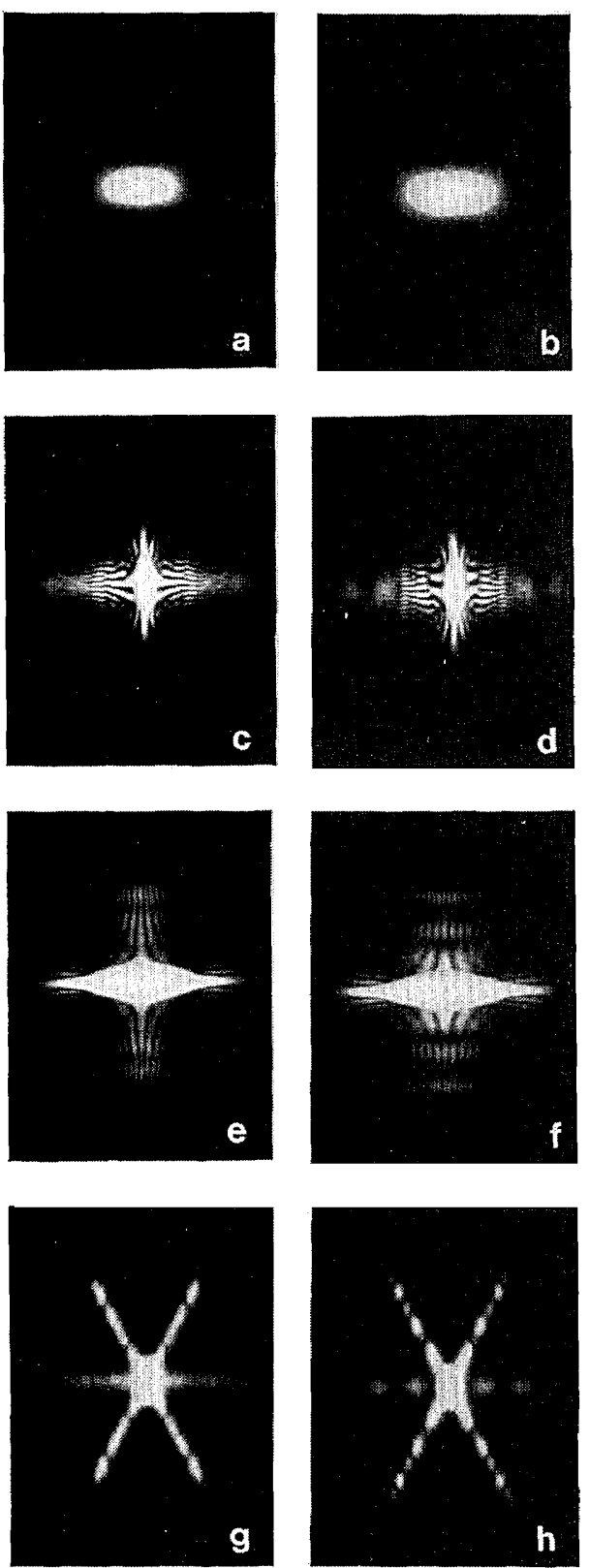

Fig. 2. Resulting averages of the four functions of the joint scheme for both the point (a, c, e, g) and the binary stars (b, d, f, h): a, b, the PF; c, d, the WD; e, f, the AF; g, h, the SP. 


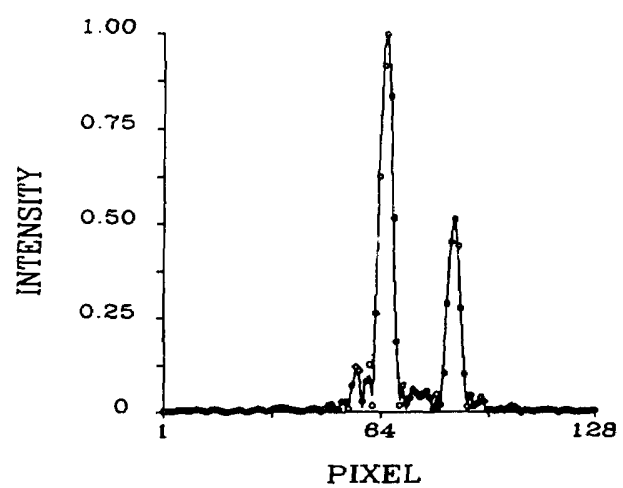

Fig. 3. Reconstruction of the binary star from the PF (solid curve) and from the SP (symbols) by the Knox-Thompson phase-integration technique.

onstrated for the SP by Knox and Thompson ${ }^{3}$ and von der Lühe. ${ }^{13}$ If that is true for $K_{i}$, then it will also be true for $P_{i}, W_{i}$, and $A_{i}$. Equations (4) and (5) implicitly denote a generalization of SSI. In fact, it is evident how to extract the average power spectrum $\left\langle\left|I_{t}(u)\right|^{2}\right\rangle$ or the autocorrelation $\left\langle Q_{t}(\Delta x)\right\rangle$ from Eqs. (5):

$$
\begin{aligned}
& \left\langle\left|I_{t}(u)\right|^{2}\right\rangle=\left\langle K_{i}(u, 0)\right\rangle=\int_{-\infty}^{+\infty} \mathrm{d} x\left\langle W_{i}(x, u)\right\rangle, \\
& \left\langle Q_{t}(\Delta x)\right\rangle=\left\langle A_{i}(\Delta x, 0)\right\rangle=\int_{-\infty}^{+\infty} \mathrm{d} x\left\langle P_{i}(x, \Delta x)\right\rangle .
\end{aligned}
$$

In other words, the autocorrelation and the power spectrum are the central row of $A_{i}$ and $K_{i}$, respectively; they can also be obtained from $P_{i}$ and $W_{i}$ by integration in $\mathrm{d} x$. It is easy to demonstrate that $K_{i}$ contains phase information for $\Delta u<u_{c}$, with $u_{c}$ being the cutoff frequency of the atmosphere. ${ }^{3,13}$ Since $K_{i}$ and $A_{i}$ are a Fourier pair, $A_{i}$ will also keep the phase information. Moreover, for the same reason, $P_{i}$ and $W_{i}$ will also implicitly contain the phase information.

From what has been reported above, there are four different possiblities to implement this generalized SSI method, preserving both high frequencies and phase. For instance, the choice of the SP would be almost equivalent to the Knox-Thompson speckle imaging technique. ${ }^{3}$ On the contrary, the PF choice seems more natural in this representation in order to avoid the computation of the FT of every single frame and thus save computing time. There are also two additional intermediate cases, which could perhaps present other advantages: the WD and the AF. Nevertheless, the disadvantages inherent to joint energy representations are that these functions are of $2 N$ dimensions, although it is always possible to develop methods to save memory. ${ }^{14}$

The joint formulation permits the unification of most speckle imaging techniques. Table 1 shows how the different methods (except triple correlation) can be applied to the averaged $\mathrm{PF},\left\langle P_{i}(x, \Delta x)\right\rangle$. Table 1 also shows the adequate kernel $\phi(\xi, \alpha)$ to define the particular representation, after Eq. (3), corresponding to each speckle technique. Note that in almost all cases, the kernel is composed of only delta functions and linear phase factors. However, since the triple correlation belongs to a third-order representation class, it is necessary to include $F^{*}(\Delta u)$ in the kernel. This renders impossible the application of the triplecorrelation technique to the $\mathrm{PF}$.

Figures 2 and 3 shows the results of a computer simulation. One hundred fifty speckle images of both a binary star and reference were generated by a standard method. ${ }^{15}$ Imaging through a 1-m telescope, a Fried parameter of $10 \mathrm{~cm}$, and a wavelength of $0.5 \mu \mathrm{m}$ were assumed. The resulting $256 \times 256$ frames were integrated in one axis to obtain one-dimensional data. The sampling interval was 5 pixels per speckle grain to avoid possible aliasing problems when computing $\Delta x /$ 2 and $\Delta u / 2$ increments. No noise was added to the frames. Figure 2 shows the results of averaging the four functions $P_{i}, W_{i}, A_{i}$, and $K_{i}$ for both the binary and the point stars. The horizontal direction corresponds to $\Delta x$ or $u$, while the vertical direction refers to $x$ or $\Delta u$, depending on the case. Figure 3 shows two reconstructions. The solid curve was obtained by first computing $\left\langle P_{i}(x, \Delta x)\right\rangle$ and then its double FT, while the symbols corresponds to direct computing of $\left\langle K_{i}(u\right.$, $\Delta u)\rangle$. In both cases the phase of the FT of the object was obtained by applying the phase-integration method of Knox-Thompson. A Wiener filtering was applied in the deconvolution. ${ }^{8}$ The two reconstructions are almost identical, and only the noise levels show small discrepancies.

One may conclude that the formulation presented here permits a generalization of methods such as standard SSI and Knox-Thompson speckle imaging. Furthermore, many other imaging methods, such as the phase gradient, are directly, and alternatively, applicable to the resulting data.

This research has been supported by the Instituto de Astrofísica de Canarias and the Comision Interministerial de Ciencia y Tecnologie.

\section{References}

1. A. Labeyrie, Astron. Astrophys. 6, 85 (1970).

2. J. C. Dainty, ed., Laser Speckle and Related Phenomena (Springer-Verlag, Berlin, 1984).

3. K. T. Knox and B. J. Thompson, Astron. J. 193, L45 (1974).

4. A. W. Lohmann, G. P. Weigelt, and B. Wirnitzer, Appl. Opt. 22, 4028 (1983).

5. G. J. M. Aitken, R. Johnson, and R. Houtman, Opt. Commun. 56, 379 (1986).

6. J. G. Walker, Opt. Acta 28, 1017 (1981).

7. J. J. Fuensalida, F. Rosa, and F. J. Fuentes, Astron. Astrophys. 191, L13 (1988).

8. R. Navarro, F. J. Fuentes, and M. Nieto-Vesperinas, Astron. Astrophys. 208, 374 (1989).

9. J. C. Dainty and J. R. Fienup, in Image Recovery, H. Stark, ed. (Academic, New York, 1987).

10. L. Cohen, J. Math. Phys. 7, 781 (1966).

11. L. D. Jacobson and H. Wechsler, Signal Processing 14, 37 (1988).

12. T. A. C. M. Claasen and W. F. G. Mecklenbrauker, Philips J. Res. 35, 217, 276, 372 (1980).

13. O. von der Lühe, J. Opt. Soc. Am. A 5, 721 (1988).

14. M. J. Northcott, G. R. Ayers, and J. C. Dainty, J. Opt. Soc. Am. A 5, 986 (1988).

15. G. R. Ayers, M. J. Northcott, and J. C. Dainty, J. Opt. Soc. Am. A 5, 963 (1988). 